\title{
Epidemiology of primary brain tumors in dr. Kariadi Hospital Semarang in 2015-2018
}

\author{
Rahmi Ardhini ${ }^{1}$ and Dodik Tugasworo ${ }^{1}$ \\ ${ }^{1}$ Medical Staff in Neurology Department Faculty of Medicine Diponegoro University/ Dr. Kariadi Hospital Semarang - Indonesia
}

\begin{abstract}
Background: Primary brain tumors are neoplasm originated from brain parenchyma and its surrounding structures. Although primary brain tumors is only $1,4 \%$ of all cancers, they causes significant morbidity and mortality. Objective: To study the epidemiology of primary brain tumors in Dr. Kariadi General Hospital Semarang between 2015 and 2018. Methods: a descriptive epidemiological study taken from medical records of hospitalized patients with primary brain tumors during $2015-2018$. The data includes demographic characteristics and clinical characteristics. Results: There were 175 suspected cases of primary brain tumor, $38.3 \%$ occurred in males and $61.7 \%$ in females. The peak incidence is in $41-50$ years $(34.3 \%) .61 .1 \%$ live in coastal areas. The frequent symptoms is headache $(44.9 \%)$, with the most common location was in the frontal lobe $(17.7 \%)$. The most common type was meningioma $(24.5 \%)$. Meningioma were more common in females $(90.7 \%)$, whereas glioma were more common in males $(60 \%)$. $46.3 \%$ patients experienced clinical improvement at the time of discharge. Conclusions: The incidence of primary brain tumors are more common in females, the peak incidence are age 41-50 years, and most of them live in coastal areas.
\end{abstract}

Keywords : epidemiology; brain tumors; primary brain tumors.

\section{Introduction}

Primary brain tumors are a group of neoplasm arising from brain parenchyma and its surrounding structures. These tumors divided into degrees of malignancy from the benign to aggressive. Although primary brain tumors only $1,4 \%$ of all cancers, they are including the most aggressive tumors which result in mortality rate of about $60 \%$. Despite these tumors, incidence are a relatively low, brain tumors cause significant health problems in worldwide by their high mortality and morbidity $[1,2]$. Benign tumor can be lethal due to their site in the brain, their ability to infiltrate locally and to transform into malignancy [3].

WHO classified central nervous system tumors based on the histological and molecular profile. By the year 2007, WHO classified based on the histopathological characteristic. And by the year of 2016, WHO renewed the diagnosis criteria by adding the molecular parameter $[4,5]$.

Primary brain tumors etiology is still unsure, but some studies investigated the risk factors such as genetic, ion radiation, viral infection, head trauma, tobacco, and alcohol. Patients with brain tumors' clinical features are based on the volume and location of the tumor, either general from the increased intracranial pressure or focal neurological symptoms [2].
The workup diagnostic for brain tumors including imaging diagnostic by CT-scan, MRI, PET scan of the brain, and cerebrospinal fluid (CSF) analysis. Contrast head MRI is the first imaging modality choice because it produces detail image of the soft tissues, but still, histopathology is the gold standard diagnosis in brain tumors. Treatments for brain tumors are conservative management to reduce intracranial pressure, surgery, radiation, and chemotherapy as indicated [6].

The importance of the brain tumors epidemiology is to know the prevalence and characteristics of brain tumors either the histological or the sociodemography of the patients. There are still limited data about brain tumors epidemiology in Indonesia so that authors study the epidemiology of primary brain tumors in RSUP dr. Kariadi Semarang. It is expected that the presence of epidemiological data can help increase knowledge and early detection of brain tumors patients can be approached.

\section{Methods}

The research was descriptive epidemiological research with retrospective approach to describe the profile of primary brain tumor patients in RSUP dr. Kariadi Semarang. Data were taken from inpatients' medical records since year 2015 to 2018. The subjects were all inpatients who diagnosed as primary brain tumors. Data

\footnotetext{
*Corresponding author: ardhini.rahmi@gmail.com
} 
that were collected were patient's demographic characteristics including gender, age, geographic region, clinical manifestation, tumors location, histopathologic features, and patient's condition at the time of discharge. Data that have been collected then analyzed descriptively to perceive the primary brain tumors' profiles.

\section{Results}

\subsection{Subjects characteristics}

There were 175 brain tumor cases suspected as primary brain tumors at RSUP dr. Kariadi Semarang between 2015-2018. All cases that suspected as primary brain tumors included in the research, including cases of unknown imaging results, no surgery, and unknown histopathological results.

The subjects consisted of 67 men (38.3\%) and 108 women $(55.1 \%)$. Subjects were divided according to age into $11.4 \%$ aged less than 20 years old, $9.1 \%$ aged $20-30$ years old, $12.6 \%$ aged $31-40$ years old, $34.3 \%$ aged 41 50 years old, $25.1 \%$ aged $51-60 \%$, and $7.4 \%$ aged more than 60 years old. The peak incidence is in age 41-50 years, that were 60 subjects $(34.3 \%)$. Based on the geographic region, $61.1 \%$ subjects were from the coast which the altitude is less than 100 meters above sea level, $22.3 \%$ subjects were from the low altitude (100$400 \mathrm{~m}$ a.s.1), and $12.6 \%$ subjects were from the high altitude ( $>400 \mathrm{~m}$ a.s.l)

Table 1. Subjects Characteristics of Primary Brain Tumor

\begin{tabular}{|c|c|c|}
\hline \multicolumn{1}{|c|}{ Variable } & n & \% \\
\hline Gender & & \\
\hline Female & 108 & 61.7 \\
\hline Male & 67 & 38.3 \\
\hline Age & & \\
\hline Less than 20 years old & 20 & 11.9 \\
\hline 20-30 years old & 16 & 11.0 \\
\hline 31-40 years old & 22 & 10.2 \\
\hline 41-50 years old & 60 & 31.4 \\
\hline 51-60 years old & 44 & 25.4 \\
\hline More than 60 years old & 13 & 10.2 \\
\hline Geographic region & & \\
\hline Coast (less than 100 m a.s.1) & 107 & 61.1 \\
\hline Low altitude (100-400 m a.s.1) & 39 & 22.3 \\
\hline $\begin{array}{l}\text { High altitude (More than 400 } \\
\text { m a.s.l) }\end{array}$ & 22 & 12.6 \\
\hline Unknown & 7 & 4.0 \\
\hline
\end{tabular}

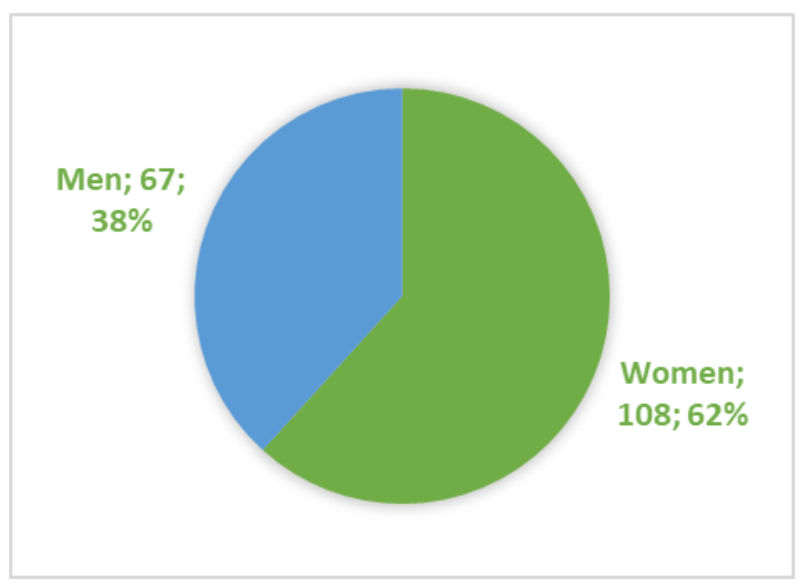

Fig. 1. Distribution of Primary Brain Tumours According to Gender

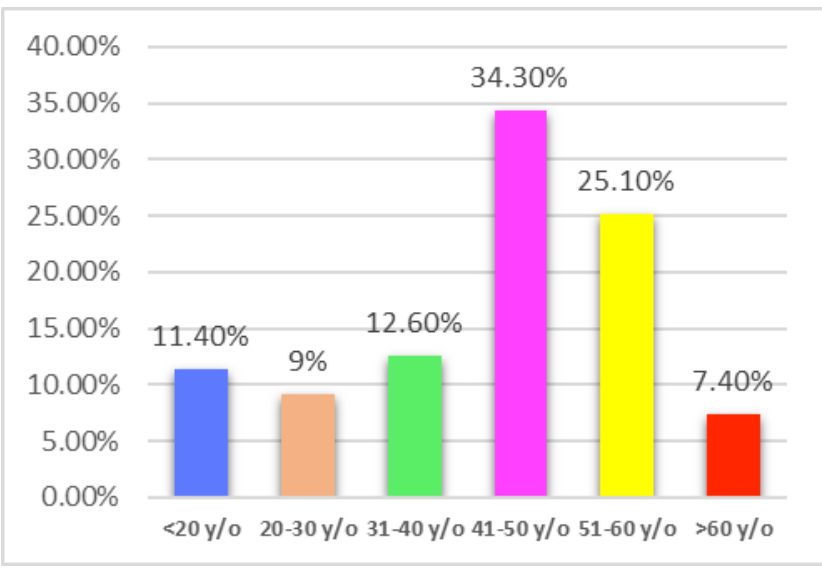

Fig. 2. Distribution of Primary Brain Tumours Based on Age

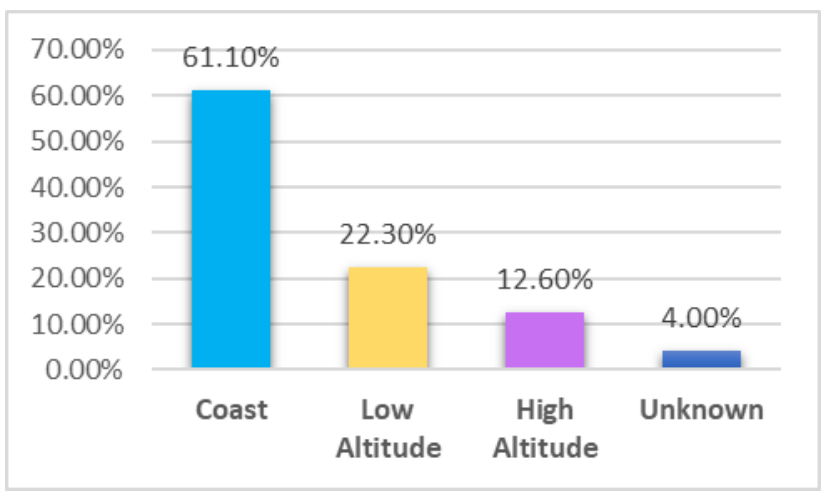

Fig. 3. Distribution of Primary Brain Based on Geographic Region

\subsection{Clinical characteristics}

The most clinical feature which was gotten from thorough history taking and physical examination was headache which was found in 142 subjects (44,.9\%). Based on the imaging of the brain, frontal lobe was the most location. As the result of the biopsy, the most common tumor according to histopathological feature 
was meningioma in 35 subjects $(20 \%)$. From all the subjects, 81 subjects $(46.3 \%)$ got clinical improvement at the time of discharge, and 36 subjects $(20.6 \%)$ passed away during hospitalization.

Table 2. Clinical Characteristics of Primary Brain Tumors

\begin{tabular}{|c|c|c|}
\hline Variable & Freq. & $\%$ \\
\hline \multicolumn{3}{|l|}{ Clinical Features } \\
\hline Headache & 142 & 44.9 \\
\hline Hemiparesis & 40 & 12.7 \\
\hline Visual Impairment & 28 & 8.9 \\
\hline Loss of Consciousness & 25 & 7.9 \\
\hline Seizure & 22 & 7.0 \\
\hline Vomiting & 14 & 4.4 \\
\hline Cranial Nerves Paresis & 13 & 4.1 \\
\hline Aphasia & 9 & 2.8 \\
\hline Dizziness & 8 & 2.5 \\
\hline Cognitive Impairment & 7 & 2.2 \\
\hline Balance Disorder & 3 & 0.9 \\
\hline \multicolumn{3}{|l|}{ Tumor Location } \\
\hline Frontal Lobe & 31 & 17.7 \\
\hline Meninges & 24 & 13.7 \\
\hline Parietal Lobe & 22 & 12.6 \\
\hline Sellar region & 21 & 12.0 \\
\hline Temporal Lobe & 14 & 8.0 \\
\hline Cerebellum & 12 & 6.9 \\
\hline Brainstem & 8 & 4.6 \\
\hline Cerebellopontine Angle & 5 & 2.9 \\
\hline Basis Cranii & 3 & 1.7 \\
\hline Ganglia Basalis & 3 & 1.7 \\
\hline Fossa Posterior & 3 & 1.7 \\
\hline Ventricle & 3 & 1.7 \\
\hline Occipital Lobe & 2 & 1.1 \\
\hline Olfactory Groove & 1 & 0.6 \\
\hline Suprainfra Tentorial & 1 & 0.6 \\
\hline Unidentified & 22 & 12.6 \\
\hline \multicolumn{3}{|l|}{ Tumor Histopathology } \\
\hline \multicolumn{3}{|l|}{$\begin{array}{l}\text { Tumors of the Neuroepithelial } \\
\text { Tissue }\end{array}$} \\
\hline $\begin{array}{l}\text { Astrocytoma WHO Gr. } 1 \\
\text { (Plomyxoid, Microcytic, } \\
\text { Gesmitocytic, Pilocytic) }\end{array}$ & 13 & 7.4 \\
\hline $\begin{array}{l}\text { Astrocytoma WHO Gr. } 2 \\
\text { (Diffuse, Fibrillary, Low- } \\
\text { grade, Oligoastrocytoma, } \\
\text { Protoplasmic) }\end{array}$ & 11 & 6.3 \\
\hline $\begin{array}{l}\text { Astrocytoma WHO Gr. } 3 \\
\text { (Anaplastic Astrocytoma) }\end{array}$ & 5 & 2.9 \\
\hline
\end{tabular}

\begin{tabular}{|c|c|c|}
\hline Glioblastoma (WHO gr. IV) & 18 & 10.3 \\
\hline Oligodendroglioma & 4 & 2.3 \\
\hline Ependymoma & 4 & 2.3 \\
\hline \multicolumn{3}{|l|}{ Tumor of the Meningen } \\
\hline $\begin{array}{l}\text { Meningioma WHO Gr.1 } \\
\text { (Microcytic, Fibrous, } \\
\text { Transitional, } \\
\text { Meningoepithelial, } \\
\text { Psammomatous, Secretory) }\end{array}$ & 35 & 20.0 \\
\hline $\begin{array}{l}\text { Meningioma WHO Gr. } 2 \\
\text { (Atypical Meningioma) }\end{array}$ & 7 & 4.0 \\
\hline $\begin{array}{l}\text { Meningioma Grade } 3 \\
\text { (Anaplastic Meningioma) }\end{array}$ & 1 & 0.6 \\
\hline \multicolumn{3}{|l|}{ Embryonal Tumor } \\
\hline Medulloblastoma & 1 & 0.6 \\
\hline Germinoma & 2 & 1.1 \\
\hline $\begin{array}{l}\text { Primitive Neuroectodermal } \\
\text { Tumour }\end{array}$ & 1 & 0.6 \\
\hline Neuroblastoma & 2 & 1.1 \\
\hline \multicolumn{3}{|l|}{$\begin{array}{l}\text { Mesenchymal, non- } \\
\text { meningothelial tumours }\end{array}$} \\
\hline Hemangioma & 3 & 1.7 \\
\hline Chordoma & 2 & 1.1 \\
\hline \multicolumn{3}{|l|}{ Tumor of the Sellar Region } \\
\hline Pituitary Adenoma & 8 & 4.6 \\
\hline \multicolumn{3}{|l|}{ Tumour of the Cranial Nerve } \\
\hline Schwannoma & 7 & 4.0 \\
\hline \multicolumn{3}{|l|}{$\begin{array}{l}\text { Lymphoma and hemopoietic } \\
\text { neoplasm }\end{array}$} \\
\hline $\begin{array}{l}\text { High-grade B cell } \\
\text { lymphoma }\end{array}$ & 1 & 0.6 \\
\hline Craniopharyngioma & 4 & 2.3 \\
\hline No Surgery & 7 & 4.0 \\
\hline $\begin{array}{l}\text { Unknown Histopathological } \\
\text { Result }\end{array}$ & 39 & 22.3 \\
\hline \multicolumn{3}{|l|}{$\begin{array}{l}\text { Condition at the time of } \\
\text { Discharged }\end{array}$} \\
\hline Getting Better & 81 & 46.3 \\
\hline Getting Worse (Palliative) & 1 & 0.6 \\
\hline Passed Away & 36 & 20.6 \\
\hline Unknown & 57 & 32.6 \\
\hline
\end{tabular}

Tumors that were more common in males were astrocytoma (55.3\%) and pituitary adenoma (62.5\%), while the more common in females was meningioma $(90.7 \%)$, hemangioma (100\%), and schwannoma (71.4\%). 


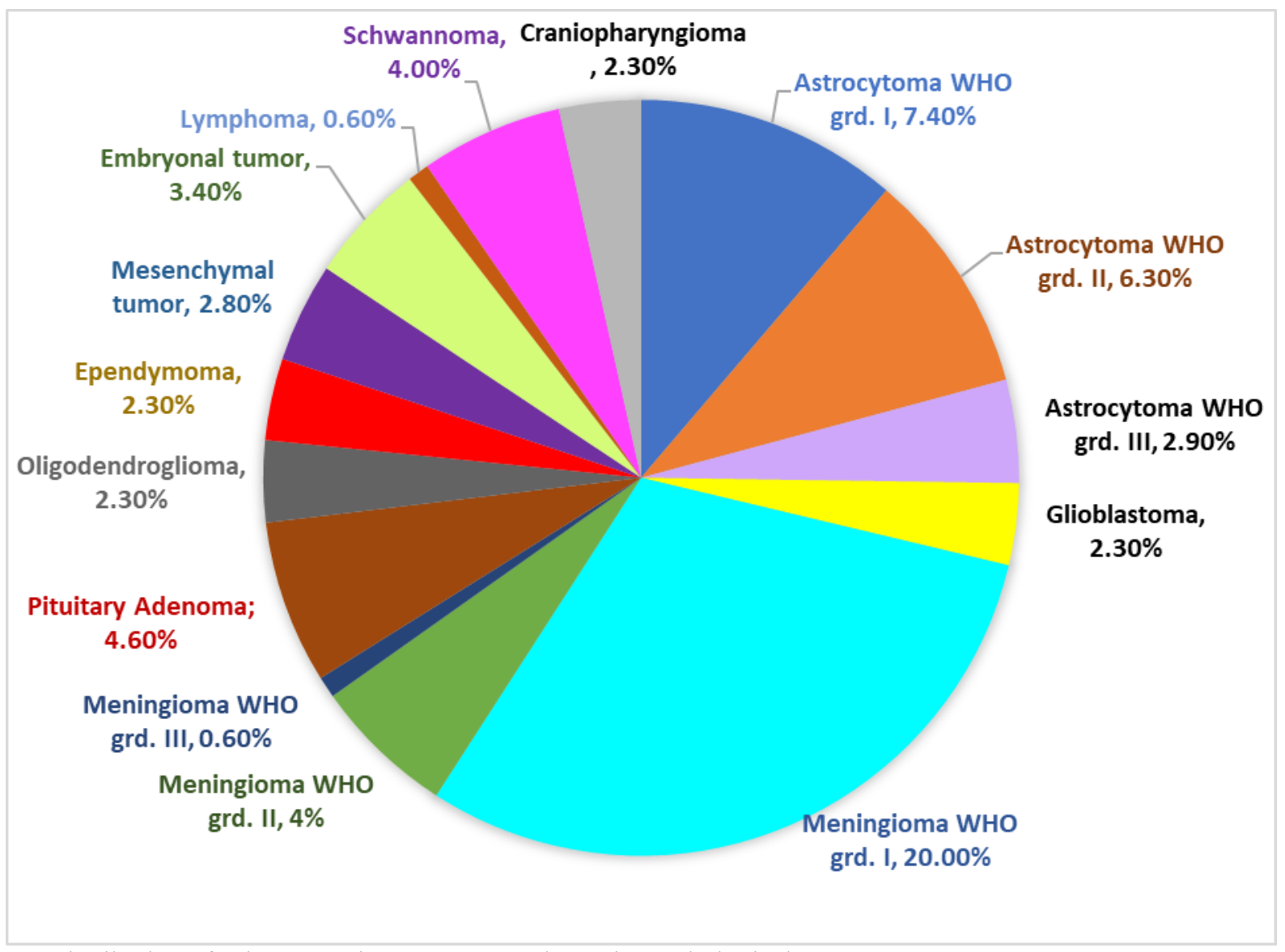

Fig. 4. Distribution of Primary Brain Tumors Based on Histopathological Features

Table 3. Tumor Histology Based on Gender

\begin{tabular}{|c|c|c|c|}
\hline \multirow{2}{*}{ Type } & \multirow{2}{*}{$\mathbf{n}$} & \multicolumn{2}{|c|}{ Gender } \\
\hline & & Men & Women \\
\hline $\begin{array}{l}\text { Astrocytoma WHO gr. I } \\
\text { (Gesmitocytic, Microcytic, } \\
\text { Pilocytic, Pilomyxoid) }\end{array}$ & 13 & $\begin{array}{c}6 \\
(46.2 \%) \\
\end{array}$ & $\begin{array}{c}7 \\
(53.8 \%) \\
\end{array}$ \\
\hline $\begin{array}{l}\text { Astrocytoma WHO gr. II } \\
\text { (Diffuse, Fibrillary, Low-grade, } \\
\text { Oligoastrocytoma, Protoplasmic) }\end{array}$ & 11 & $\begin{array}{c}9 \\
(81.8 \%)\end{array}$ & $\begin{array}{c}2 \\
(18.2 \%)\end{array}$ \\
\hline $\begin{array}{l}\text { Astrocytoma WHO gr. III } \\
\text { (Anaplastic) }\end{array}$ & 5 & $\begin{array}{c}1 \\
(20 \%)\end{array}$ & $\begin{array}{c}4 \\
(80 \%) \\
\end{array}$ \\
\hline Glioblastoma (WHO gr. IV) & 14 & $\begin{array}{c}8 \\
(57.1 \%) \\
\end{array}$ & $\begin{array}{c}6 \\
(42.9 \%) \\
\end{array}$ \\
\hline Oligodendroglioma & 5 & $\begin{array}{c}4 \\
(80 \%)\end{array}$ & $\begin{array}{c}1 \\
(20 \%)\end{array}$ \\
\hline Ependymoma & 3 & $\begin{array}{c}2 \\
(66.7 \%) \\
\end{array}$ & $\begin{array}{c}1 \\
(33.3 \%) \\
\end{array}$ \\
\hline $\begin{array}{l}\text { Meningioma WHO grd. I } \\
\text { (Meningothelial, Microcytic, } \\
\text { Psammomatous, Secretory, } \\
\text { Transitional) }\end{array}$ & 10 & $\begin{array}{c}1 \\
(10 \%)\end{array}$ & $\begin{array}{c}9 \\
(90 \%)\end{array}$ \\
\hline Medulloblastoma & 1 & $\begin{array}{c}1 \\
(100 \%) \\
\end{array}$ & \\
\hline Germinoma & 2 & $\begin{array}{c}1 \\
(50 \%) \\
\end{array}$ & $\begin{array}{c}1 \\
(50 \%) \\
\end{array}$ \\
\hline $\begin{array}{l}\text { Primitive Neuroectodermal } \\
\text { Tumour (PNET) }\end{array}$ & 1 & & $\begin{array}{c}1 \\
(100 \%)\end{array}$ \\
\hline Neuroblastoma & 1 & & $\begin{array}{c}1 \\
(100 \%) \\
\end{array}$ \\
\hline
\end{tabular}




\begin{tabular}{|c|c|c|c|}
\hline Hemangioma & 3 & & $\begin{array}{c}3 \\
(100 \%)\end{array}$ \\
\hline Chordoma & 2 & $\begin{array}{c}1 \\
(50 \%)\end{array}$ & $\begin{array}{c}1 \\
(50 \%)\end{array}$ \\
\hline Pituitary Adenoma & 6 & $\begin{array}{c}3 \\
(50 \%)\end{array}$ & $\begin{array}{c}3 \\
(50 \%)\end{array}$ \\
\hline Schwannoma & 1 & & $\begin{array}{c}1 \\
(100 \%)\end{array}$ \\
\hline Primary CNS lymphoma & 1 & $\begin{array}{c}1 \\
(100 \%)\end{array}$ & \\
\hline No Surgery & 6 & $\begin{array}{c}2 \\
(33.3 \%)\end{array}$ & $\begin{array}{c}4 \\
(66.7 \%)\end{array}$ \\
\hline $\begin{array}{l}\text { Unknown Histopathological } \\
\text { Result }\end{array}$ & 33 & $\begin{array}{c}13 \\
(39.4 \%)\end{array}$ & $\begin{array}{c}20 \\
(60.6 \%)\end{array}$ \\
\hline TOTAL & & 53 & 65 \\
\hline
\end{tabular}

Meningioma more common in 41-50 years $(55.8 \%)$.

Table 4. Tumor Histology According to Age

\begin{tabular}{|c|c|c|c|c|c|c|c|}
\hline \multirow{2}{*}{ Type } & \multirow{2}{*}{$\mathbf{n}$} & \multicolumn{6}{|c|}{ Age (years old) } \\
\hline & & $<\mathbf{2 0}$ & 21-30 & $31-40$ & 41-50 & $51-60$ & $>60$ \\
\hline $\begin{array}{l}\text { Astrocytoma WHO gr. I } \\
\text { (Gesmitocytic, Microcytic, } \\
\text { Pilocytic, Pilomyxoid) }\end{array}$ & 13 & $\begin{array}{c}1 \\
(7.7 \%)\end{array}$ & $\begin{array}{c}3 \\
(23.1 \%)\end{array}$ & & $\begin{array}{c}6 \\
(46.2 \%)\end{array}$ & $\begin{array}{c}1 \\
(7.7 \%)\end{array}$ & $\begin{array}{c}2 \\
(15.4 \%)\end{array}$ \\
\hline $\begin{array}{l}\text { Astrocytoma WHO gr. II } \\
\text { (Diffuse, Fibrillary, Low-grade, } \\
\text { Oligoastrocytoma, } \\
\text { Protoplasmic) }\end{array}$ & 11 & $\begin{array}{c}1 \\
(9.1 \%)\end{array}$ & $\begin{array}{c}2 \\
(18.2 \%)\end{array}$ & $\begin{array}{c}2 \\
(18.2 \%)\end{array}$ & $\begin{array}{c}1 \\
(9.1 \%)\end{array}$ & $\begin{array}{c}5 \\
(45.5 \%)\end{array}$ & \\
\hline $\begin{array}{l}\text { Astrocytoma WHO gr. III } \\
\text { (Anaplastic) }\end{array}$ & 5 & & & & $\begin{array}{c}2 \\
(40 \%) \\
\end{array}$ & $\begin{array}{c}2 \\
(40 \%)\end{array}$ & $\begin{array}{c}1 \\
(20 \%)\end{array}$ \\
\hline Glioblastoma (WHO gr. IV) & 18 & $\begin{array}{c}1 \\
(5.6 \%)\end{array}$ & $\begin{array}{c}2 \\
(11.1 \%)\end{array}$ & & $\begin{array}{c}6 \\
(33.3 \%)\end{array}$ & $\begin{array}{c}8 \\
(44.4 \%)\end{array}$ & $\begin{array}{c}1 \\
(5.6 \%)\end{array}$ \\
\hline Oligodendroglioma & 4 & & & & $\begin{array}{c}3 \\
(75 \%)\end{array}$ & $\begin{array}{c}1 \\
(25 \%)\end{array}$ & \\
\hline Ependymoma & 4 & $\begin{array}{c}3 \\
(75 \%) \\
\end{array}$ & $\begin{array}{c}1 \\
(25 \%)\end{array}$ & & & & \\
\hline $\begin{array}{l}\text { Meningioma WHO grd. I } \\
\text { (Meningothelial, Microcytic, } \\
\text { Psammomatous, Secretory, } \\
\text { Transitional) }\end{array}$ & 35 & & $\begin{array}{c}1 \\
(2.9 \%)\end{array}$ & $\begin{array}{c}3 \\
(8.6 \%)\end{array}$ & $\begin{array}{c}21 \\
(60 \%)\end{array}$ & $\begin{array}{c}8 \\
(22.9 \%)\end{array}$ & $\begin{array}{c}2 \\
(5.7 \%)\end{array}$ \\
\hline $\begin{array}{l}\text { Meningioma WHO grd. II } \\
\text { (Atypical) }\end{array}$ & 7 & & $\begin{array}{c}1 \\
(14.3 \%)\end{array}$ & $\begin{array}{c}2 \\
(28.6 \%)\end{array}$ & $\begin{array}{c}3 \\
(42.9 \%)\end{array}$ & $\begin{array}{c}1 \\
(14.3 \%)\end{array}$ & \\
\hline $\begin{array}{l}\text { Meningioma WHO grd. III } \\
\text { (Anaplastic) }\end{array}$ & 1 & & & $\begin{array}{c}1 \\
(100 \%)\end{array}$ & & & \\
\hline Medulloblastoma & 1 & $\begin{array}{c}1 \\
(100 \%)\end{array}$ & & & & & \\
\hline Germinoma & 2 & $\begin{array}{c}2 \\
(100 \%)\end{array}$ & & & & & \\
\hline $\begin{array}{l}\text { Primitive Neuroectodermal } \\
\text { Tumour (PNET) }\end{array}$ & 1 & $\begin{array}{c}1 \\
(100 \%)\end{array}$ & & & & & \\
\hline Neuroblastoma & 2 & $\begin{array}{c}1 \\
(50 \%)\end{array}$ & & & $\begin{array}{c}1 \\
(50 \%)\end{array}$ & & \\
\hline Hemangioma & 3 & $\begin{array}{c}1 \\
(33.3 \%) \\
\end{array}$ & $\begin{array}{c}1 \\
(33.3 \%) \\
\end{array}$ & & & & $\begin{array}{c}1 \\
(33.3 \%)\end{array}$ \\
\hline Chordoma & 2 & & $\begin{array}{c}1 \\
(50 \%)\end{array}$ & & $\begin{array}{c}1 \\
(50 \%)\end{array}$ & & \\
\hline Pituitary Adenoma & 8 & & & 2 & 3 & 3 & \\
\hline
\end{tabular}




\begin{tabular}{|c|c|c|c|c|c|c|c|}
\hline & & & & $(25 \%)$ & $(37.5 \%)$ & $(37.5 \%)$ & \\
\hline Schwannoma & 7 & $\begin{array}{c}1 \\
(14.3 \%)\end{array}$ & & $\begin{array}{c}3 \\
(42.9 \%)\end{array}$ & $\begin{array}{c}1 \\
(14.3 \%)\end{array}$ & $\begin{array}{c}2 \\
(28.6 \%)\end{array}$ & \\
\hline Primary CNS lymphoma & 1 & & & & & $\begin{array}{c}1 \\
(100 \%)\end{array}$ & \\
\hline Craniopharyngioma & 4 & $\begin{array}{c}3 \\
(75 \%) \\
\end{array}$ & & & $\begin{array}{c}1 \\
(25 \%)\end{array}$ & & \\
\hline No Surgery & 7 & & $\begin{array}{c}1 \\
(14.3 \%)\end{array}$ & $\begin{array}{c}1 \\
(14.3 \%)\end{array}$ & $\begin{array}{c}1 \\
(14.3 \%)\end{array}$ & $\begin{array}{c}2 \\
(28.6 \%)\end{array}$ & $\begin{array}{c}2 \\
(28.6 \%)\end{array}$ \\
\hline $\begin{array}{l}\text { Unknown Histopathological } \\
\text { Result }\end{array}$ & 39 & $\begin{array}{c}4 \\
(10.3 \%) \\
\end{array}$ & $\begin{array}{c}3 \\
(7.7 \%) \\
\end{array}$ & $\begin{array}{c}8 \\
(20.5 \%) \\
\end{array}$ & $\begin{array}{c}10 \\
(25.6 \%)\end{array}$ & $\begin{array}{c}10 \\
(25.6 \%) \\
\end{array}$ & $\begin{array}{c}4 \\
(10.3 \%) \\
\end{array}$ \\
\hline TOTAL & & 20 & 16 & 22 & 60 & 44 & 13 \\
\hline
\end{tabular}

Subjects with primary brain tumors mostly lived in coastal area, i.e. astrocytoma (57.4\%), meningioma $(60.4 \%)$, schwannoma $(71.4 \%)$, and craniopharyngioma $(50 \%)$. While all subjects with ependymoma, coastal area. One subject with primary CNS lymphoma and medulloblastoma lived in the low altitude region. None of the tumors were from high altitude regions. hemangioma, germinoma, and pituitary adenoma lived in

Table 5. Tumor Types Frequency According to The Altitude of Residence

\begin{tabular}{|c|c|c|c|c|c|}
\hline \multirow{2}{*}{ Type } & \multirow{2}{*}{$\mathbf{n}$} & \multicolumn{4}{|c|}{ Altitude of Residence } \\
\hline & & Coast & Low Altitude & High Altitude & Unknown \\
\hline $\begin{array}{l}\text { Astrocytoma WHO gr. I } \\
\text { (Gesmitocytic, Microcytic, } \\
\text { Pilocytic, Pilomyxoid) }\end{array}$ & 13 & $\begin{array}{c}8 \\
(61.5 \%)\end{array}$ & $\begin{array}{c}3 \\
(23.1 \%)\end{array}$ & $\begin{array}{c}2 \\
(15.4 \%)\end{array}$ & \\
\hline $\begin{array}{l}\text { Astrocytoma WHO gr. II } \\
\text { (Diffuse, Fibrillary, Low-grade, } \\
\text { Oligoastrocytoma, } \\
\text { Protoplasmic) }\end{array}$ & 11 & $\begin{array}{c}8 \\
(72.7 \%)\end{array}$ & $\begin{array}{c}1 \\
(9.1 \%)\end{array}$ & $\begin{array}{c}2 \\
(18.2 \%)\end{array}$ & \\
\hline $\begin{array}{l}\text { Astrocytoma WHO gr. III } \\
\text { (Anaplastic) }\end{array}$ & 5 & $\begin{array}{c}3 \\
(60 \%) \\
\end{array}$ & $\begin{array}{c}1 \\
(20 \%) \\
\end{array}$ & $\begin{array}{c}1 \\
(20 \%) \\
\end{array}$ & \\
\hline Glioblastoma (WHO gr. IV) & 18 & $\begin{array}{c}8 \\
(44.4 \%) \\
\end{array}$ & $\begin{array}{c}6 \\
(33.3 \%) \\
\end{array}$ & $\begin{array}{c}3 \\
(16.7 \%) \\
\end{array}$ & $\begin{array}{c}1 \\
(5.6 \%) \\
\end{array}$ \\
\hline Oligodendroglioma & 4 & $\begin{array}{c}2 \\
(50 \%)\end{array}$ & $\begin{array}{c}1 \\
(25 \%)\end{array}$ & $\begin{array}{c}1 \\
(25 \%)\end{array}$ & \\
\hline Ependymoma & 4 & $\begin{array}{c}3 \\
(75 \%) \\
\end{array}$ & & & $\begin{array}{c}1 \\
(25 \%)\end{array}$ \\
\hline $\begin{array}{l}\text { Meningioma WHO grd. I } \\
\text { (Meningothelial, Microcytic, } \\
\text { Psammomatous, Secretory, } \\
\text { Transitional) }\end{array}$ & 35 & $\begin{array}{c}22 \\
(62.9 \%)\end{array}$ & $\begin{array}{c}8 \\
(22.9 \%)\end{array}$ & $\begin{array}{c}4 \\
(11.4 \%)\end{array}$ & $\begin{array}{c}1 \\
(2.9 \%)\end{array}$ \\
\hline $\begin{array}{l}\text { Meningioma WHO grd. II } \\
\text { (Atypical) }\end{array}$ & 7 & $\begin{array}{c}3 \\
(42.9 \%) \\
\end{array}$ & $\begin{array}{c}1 \\
(14.3 \%)\end{array}$ & $\begin{array}{c}2 \\
(28.6 \%)\end{array}$ & $\begin{array}{c}1 \\
(14.3 \%)\end{array}$ \\
\hline $\begin{array}{l}\text { Meningioma WHO grd. III } \\
\text { (Anaplastic) }\end{array}$ & 1 & $\begin{array}{c}1 \\
(100 \%)\end{array}$ & & & \\
\hline Medulloblastoma & 1 & & $\begin{array}{c}1 \\
(100 \%)\end{array}$ & & \\
\hline Germinoma & 2 & $\begin{array}{c}2 \\
(100 \%) \\
\end{array}$ & & & \\
\hline $\begin{array}{l}\text { Primitive Neuroectodermal } \\
\text { Tumour (PNET) }\end{array}$ & 1 & $\begin{array}{c}1 \\
(100 \%)\end{array}$ & & & \\
\hline Neuroblastoma & 2 & $\begin{array}{c}1 \\
(50 \%) \\
\end{array}$ & & & $\begin{array}{c}1 \\
(50 \%) \\
\end{array}$ \\
\hline Hemangioma & 3 & $\begin{array}{c}3 \\
(100 \%) \\
\end{array}$ & & & \\
\hline Chordoma & 2 & $\begin{array}{c}1 \\
(50 \%) \\
\end{array}$ & $\begin{array}{c}1 \\
(50 \%) \\
\end{array}$ & & \\
\hline Pituitary Adenoma & 8 & $\begin{array}{c}8 \\
(100 \%) \\
\end{array}$ & & & \\
\hline Schwannoma & 7 & 5 & & 1 & 1 \\
\hline
\end{tabular}




\begin{tabular}{|l|c|c|c|c|c|}
\hline & & $(71.4 \%)$ & & $(14.3 \%)$ & $(14.3 \%)$ \\
\hline Primary CNS lymphoma & 1 & & $\begin{array}{c}1 \\
(100 \%)\end{array}$ & & 1 \\
\hline Craniopharyngioma & 4 & $\begin{array}{c}2 \\
(50 \%)\end{array}$ & $\begin{array}{c}1 \\
(25 \%)\end{array}$ & $\begin{array}{c}1 \\
(25 \%)\end{array}$ \\
\hline No Surgery & 7 & $\begin{array}{c}4 \\
(57.1 \%)\end{array}$ & $\begin{array}{c}1 \\
(14.3 \%)\end{array}$ & $\begin{array}{c}2 \\
(28.6 \%)\end{array}$ & \\
\hline $\begin{array}{l}\text { Unknown Histopathological } \\
\text { Result }\end{array}$ & 39 & $\begin{array}{c}22 \\
(56.4 \%)\end{array}$ & $\begin{array}{c}13 \\
(33.3 \%)\end{array}$ & $\begin{array}{c}4 \\
(10.3 \%)\end{array}$ & \\
\hline TOTAL & & 107 & 39 & 22 & 7 \\
\hline
\end{tabular}

\section{Discussion}

This research was descriptive epidemiological research with primary brain tumors patients who were treated in RSUP dr. Kariadi Semarang year $2015-2018$ as the subjects by retrospective approach from patients' medical records. In this research, female incidence was more common than male, i.e. $38.3 \%$ male and $61.7 \%$ female, concordant to the older references from the study in Spain and Korea which the frequency of women was higher than men, i.e. $57.25 \%$ and $61.4 \%$, while the database from CBTRUS and meta-analysis study in America claimed that the incidence of female was more than men which were 24.77 vs 20.34 per 100.000 populations and 15.8 vs 14.33 per 100.000 populations. However, Pineros et al. claimed from the recent study that the incidence of male was equal to female $[1,7,10$, 13, 14].

Primary brain tumor patients were mostly at the age of 41-50 years old, which was different from other recent studies that primary brain tumor's incidence increased by the age, especially high-grade glioma which often found at the age of more than 60 years old [7-9].

The most common symptoms were headache concordant to the recent studies in which headache was the most common symptom of either primary or metastatic brain tumors as the result of increased intracranial pressure. Adams et al. claimed that the incidence of headache was $48-56 \%[11,12]$.

In this research, brain tumors most were in frontal lobe, while some recent studies claimed meninges as the most location of brain tumors wherefor in this research, the incidence of axial tumors e.g. astrocytoma was more than the tumors of meninges [7].

The most histopathological feature in this research was meningioma (24.5\%). There were various results of the recent studies, the studies from RSCM, Pineros et al. from America, and Fuentes-Raspall et al. from Spain claimed that the most histopathological feature was glioma, while CBTRUS claimed that the most common primary brain tumor was meningioma. Some studies showed that the incidence of meningioma was higher in western than in Asia $[7,10,15,16]$.

Most patients got better at the time of discharged, but because of the data limitations, factors affecting the outcomes were not analyzed.

Most common primary brain tumors in this study are meningioma $(24.5 \%)$, similar to the literature that showed the ratio of male: female were 1:1.4-2.6 [16].
Glioma was found more in men than women $55.8 \%$ vs $44.2 \%$ ) which concordant to the recent references and studies. Sun et al. claimed that there was oncogenic and genetic mutation mechanism which is higher in men so that the prevalence of glioma is higher in men than women $[7,17,18]$.

Data on environmental exposure to primary brain tumors remain unknown and controversial. In this research, the geographic region was categorized based on Samodra et al. Most patients with primary brain tumors lived in the coastal areas $(<100 \mathrm{~m}$ a.s.l). There were no studies that analyzed the altitude of residence to primary brain tumors. In this research, $61.14 \%$ patients lived in the coastal areas, but there was no data about the environmental and occupational exposures. In Indonesia which is an agrarian country, almost all residents work as a farmer. According to Badan Pusat Statistik (BPS), populations aged $>15$ years old who work in agricultural is about $38.35 \%$. This fact proves that there is high exposure to agricultural chemicals such as pesticides that are believed to be neurotoxic and carcinogenic. A metaanalysis study in 1998 by Kuder et al showed a moderately increased risk that was statistically significant for primary brain tumors among farmworkers. Otherwise, coastal area also has more pollution from industry and vehicles than the highland $[2,19-21]$.

\section{Conclusion}

There were 175 cases of brain tumors suspected as primary brain tumors in RSUP dr. Kariadi Semarang between 2015-2018. The incidence more common in females than males, which most in the age of 41-50 years old, and almost all lived in the coastal areas. The most common clinical feature is headache caused by space-occupying effect and increased intracranial pressure. The most type of tumor according to histopathological feature is meningioma, which found more common in female. Patients mostly live on the coast where there is agricultural chemicals exposure as one of the brain tumor risk factors.

\section{References}

1. P. de Robles, K.M. Fiest, A.D. Frolkis, T. Pringsheim, C. Atta, C. St.Germaine-Smith, et al,. Worldwide incidence and prevalence of primary brain tumors: a systematic review and metaanalysis. J. Neurooncology 17(6) (2015) 
2. M.J. Strong, J. Garces, J.C. Vera, M. Mathkour, N. Emerson, M.L. Ware, Brain tumors: epidemiology and current trends in treatment. J. Brain tumors neurooncol 1 (2015)

3. P.A. McKinney, Brain tumors: incidence, survival, and aetiology, J. Neurol Neurosurg Psychiatry 75 (2004)

4. D.N. Louis, H. Ohgaki, O.D. Wiestler, W.K. Cavenee, P.C. Burger, A. Jouvet, et al., The 2007 WHO Classification of Tumours of the Central Nervous System, Acta Neuropathol 114 (2007)

5. D.N. Louis, A. Perry, G. Reifenberger, A. von Deimling, D. Figarella-Branger, W.K. Cavenee, et al., The 2016 World Health Organization Classification of Tumors of the Central Nervous System: a summary, Acta Neuropathol 131 (2016)

6. T. Aninditha, T.A.S. Ranakusuma, Tumor otak primer, In: Aninditha T, Wiratman W. Buku Ajar Neurologi. Departemen Neurologi FKUI. Jakarta: Penerbit Kedokteran Indonesia (2017)

7. Q.T. Ostrom, H. Gittleman, P. Liao, T. Vecchione-Koval, Y. Wolinsky, C. Kruchko, et al., CBTRUS Statistical Report: Primary brain and other central nervous system tumors diagnosed in the United States in 2010-2014, J. Neurooncology 19 (2017)

8. H.B Newton, Overview of brain tumor epidemiology, In: H.B. Newton HB, ed. Handbook of neurooncology neuroimaging. Second edition. (Elsevier, London, 2016)

9. R. El-Zein, M. Bondy, M. Wrensch. Epidemiology of brain tumors, Humana Press Inc., New Jersey, (2005)

10. M. Pineros, M.S. Sierra, M.I. Izarzugaza, D. Forman. Descriptive epidemiology of brain and central nervous system cancers in Central and South America, Cancer epidemiology 44S (2016)

11. C. Adams, J. Sullivan, T.W. Vitaz, Clinical presentation of brain tumors, Available from: http://www.intechopen.com
12. S. Ranjan, D. Schiff, Headache as complication of cancer, In: D. Schiff, I. Arrillaga, P.Y. Wen, Ed. Cancer neurology in clinical practice. $3^{\text {rd }} \mathrm{Ed}$. (Springer, Switzerland, 2018)

13. R. Fuentes-Raspall, M. Solans, A. Roca-Barcelo, L. Vilardell, M. Puigdemont, S. del Barco, et al. Descriptive epidemiology of primary malignant and non-malignant central nervous tumors in Spain: Results from the Girona Cancer Registry (1994-2013), Cancer epidemiology (2017)

14. K.W. Jung, J. Ha, S.H. Lee, YJ. Won, H. Yoo, An update nationwide epidemiology of primary brain tumors in Republic of Korea, Brain tumor res treat 1 (2013)

15. R.A. Primandari, Gambaran gangguan fungsi kognitif pada tumor otak primer dan metastasis, Tesis (2016)

16. B.J. Park, H.K. Kim, B. Sade, J.H. Lee, Epidemiology, In: J.H. Lee, Ed. Meningiomas, Springer-Verlag, London (2008)

17. Q.T. Ostrom, H. Gittleman, L. Stetson, S.M. Virk, J.S. Barnholtz-Sloan, Epidemiology of gliomas, In: Raizer J, Parsa A, Ed. Current understanding and treatment of gliomas (Springer Int Pub, Switzerland, 2015)

18. T. Sun, A. Plutynski, S. Ward, J.B. Rubin, An Integrative view on sex differences in brain tumors, Cell Mol Life Sci (2015)

19. T.B. Samodra, M. Santosa, Pola penghunian dalam transformasi altitude dan kontribusinya dalam sistim ventilasi rumah tinggal pedesaan, Seminar Nasional (2006)

20. Badan Pusat Statistik, Keadaan angkatan kerja di Indonesia: Agustus 2010, Katalog BPS. Jakarta. (2011)

21. J. Gomes, A.A. Zayadi, A.. Guzman, Occupational and environmental risk factors of adult primary brain cancers: a systematic review, Int. J. of Occupational Env Med. 2 (2011) 